\title{
ONCOGENICIDADE DO PAPILOMAVÍRUS HUMANO E 0 GRAU DE NEOPLASIA INTRA-EPITELIAL ANAL EM DOENTES HIV POSITIVO
}

\author{
Carmen Ruth Manzione, Sidney Roberto Nadal*, Edenilson Eduardo Calore \\ Trabalho realizado pela Equipe de Proctologia e pela Seção de Patologia do \\ Instituto de Infectologia Emilio Ribas, São Paulo, SP.
}

RESUMO - OBjEtIVo. Avaliar se o grau de neoplasia intra-epitelial anal (NIA) está associado ao tipo do HPV em doentes HIV positivo, já que esses apresentam imunodepressão durante longos períodos.

Métodos. Identificamos os tipos do HPV, pelo método da reação em cadeia da polimerase (PCR), e realizamos exame anatomopatológico para avaliar o grau de NIA em 39 homens HIV positivo portadores de condilomas acuminados perianais.

Resultados. Observamos NIA de alto grau em nove $(23,1 \%)$ e NIA de baixo grau em 30 doentes $(\mathbf{7 6 , 9 \% )}$. Os tipos virais mais observados foram os não oncogênicos 6 e II em $64 \%$ e os oncogênicos 16 , 18 e 31 em $20,5 \%$. Não identificamos o tipo viral em quatro doentes $(10,2 \%)$, embora 0 teste revelasse a presença do DNA viral. Comparando o padrão histológico e os tipos virais, observamos que os tipos não oncogênicos do HPV também podem estar associados ao desenvolvimento de NIA de alto grau.

Conclusäo. Os resultados obtidos nas condições de execução deste estudo permitem concluir que tanto os tipos oncogênicos como os não oncogênicos de HPV podem estar associados ao desenvolvimento de NIA de alto grau em doentes HIV positivo.

UnITERMOS: AIDS. Infecção pelo papilomavírus humano. Neoplasia intra-epitelial anal. PCR. Condiloma acuminado perianal. Câncer anal.

\section{INTRODUÇÃO}

Dentre os vários agentes etiológicos que provocam doenças na região perianal de indivíduos HIV positivo, o papilomavírus humano (HPV) é o mais comum. A maioria das infecções pelo HPV não tem qualquer conseqüência clínica, mas cerca de $10 \%$ dos pacientes desenvolverão verrugas, papilomas ou displasias'. É também descrita a possibilidade de progressão de carcinoma "in situ" para invasor $^{2}$ e a maioria ocorreria na zona de transição do canal anal ${ }^{3}$.

O vírus, por si, não é suficiente para a carcinogênese, e a progressão tumoral, que ocorre em pequena porcentagem de indivíduos, pode ser estimulada por mutágenos químicos ou físicos'. Os fatores ligados ao desenvolvimento da neoplasia intra-epitelial anal (NIA) e à incidência elevada de recidivas nesses doentes não estão ainda completamente esclarecidos e a prevalência do HPV em lesões anogenitais de doentes HIV positivo não é suficiente para explicar a grande proporção de displasias neste grupo de enfermos ${ }^{4}$.

\footnotetext{
* Correspondência:

R. Dr. Virgílio Carvalho Pinto, 381 - Ap. 23 CEP: 05415-030 - São Paulo - SP
}

Vários fatores estão associados à maior probabilidade de desenvolvimento de displasia nos condilomas acuminados perianais: prática de sexo anal e soropositividade para HIV ${ }^{58}$, imunodepressãa ${ }^{9}$, fases avançadas da infecção pelo $H V^{10}$, lesões verrucosas acima da linha pectínea ${ }^{7}$, contagens de linfócitos T CD4 inferiores a $500 / \mathrm{mm}^{3}$ 9,11-13 e tipo viral do HPVI,14. Entretanto, o tempo de aparecimento dos condilomas, tratamento tópico anterior e associação com outras doenças sexualmente transmissíveis não representaram fatores de risco ${ }^{7}$.

Dos mais de 80 tipos de HPV, 30 incidem no trato anogenital ${ }^{15}$. A infecção pelos tipos 16 e 18 é a mais associada ao câncer e displasia do colo uterino. Os tipos 31, 33 e 35 têm risco médio para câncer e os tipos 6 e II podem causar displasia leve, a exemplo do descrito para 0 trato genital ${ }^{5,7,16}$. Estas observações impõem a necessidade de conhecer o tipo do HPV para o controle efetivo dos doentes portadores de condilomas perianais.

Todavia, alguns autores ${ }^{17-19}$ relataram que a imunidade talvez seja o fator mais importante no desenvolvimento de displasias e neoplasias. Desta forma, resolvemos avaliar se o grau de NIA está associado ao tipo do HPV em doentes HIV positivo, já que esses apresentam imunodepressão durante longos períodos.

\section{MÉtodos}

Identificamos os tipos do HPV em 39 doentes, dentre os portadores de condilomas acuminados perianais que operamos entre agosto de 94 a dezembro de 99 no Serviço de Proctologia do Instituto de Infectologia Emílio Ribas. O estudo é retrospectivo. Incluímos todos os doentes operados nesse período e cujos blocos de parafina foram encontrados. Todos eram homossexuais masculinos, soropositivos para o HIV, com média etária de 32 anos e extremos de 18 e 64 anos, sem doença oportunista associada e com contagens de linfócitos T CD4+ entre 200 e 500/ $/ \mathrm{mm}^{3}$.

Enviamos todos os condilomas extirpados para exame anatomopatológico, para avaliar o grau de NIA. Um de nós (EEC) examinou as lâminas de todos os doentes em microscópio óptico para constatar a presença e a gravidade das lesões características.

Firmou-se o diagnóstico histológico de infecção pelo HPV pelo achado de coilocitose (células com grandes vacúolos perinucleares), disceratose (ceratinização imperfeita das células epidérmicas) e discariose (aumento e irregularidade na forma dos núcleos e hipercromatismo $)^{20}$.

Consideramos displasia leve a existência de anormalidades celulares como aumento do 
Tabela I - Distribuição de 39 doentes HIV + operados de condilomas acuminados anais, segundo o tipo do HPV. Instituto de Infectologia Emílio Ribas, São Paulo, 2003

\begin{tabular}{ccc}
\hline & \multicolumn{2}{c}{ Doentes } \\
\cline { 2 - 3 } Tipos de HPV & $n$ & $\%$ \\
6 & 10 & 25,6 \\
11 & 15 & 38,5 \\
16 & 02 & 5,1 \\
18 & 01 & 2,6 \\
31 & 05 & 12,8 \\
53 & 02 & 5,1 \\
nãotipável & 04 & 10,3 \\
Total & 39 & 100 \\
\hline
\end{tabular}

\begin{tabular}{|c|c|c|c|c|c|c|}
\hline \multirow[t]{2}{*}{ Tipos de HPV } & \multicolumn{2}{|c|}{ NIAA } & \multicolumn{2}{|c|}{ NIAB } & \multicolumn{2}{|c|}{ total } \\
\hline & $n$ & $\%$ & $n$ & $\%$ & $n$ & $\%$ \\
\hline $\begin{array}{c}6 \\
11 \\
16 \\
18 \\
31 \\
53 \\
\text { não tipável }\end{array}$ & $\begin{array}{l}03 \\
02 \\
00 \\
01 \\
01 \\
01 \\
01\end{array}$ & $\begin{array}{c}(33,3) \\
(22,2) \\
02 \\
(11,1) \\
(11,1) \\
(11,1) \\
(11,1)\end{array}$ & $\begin{array}{c}07 \\
13 \\
(6,7) \\
00 \\
04 \\
01 \\
03\end{array}$ & $\begin{array}{c}(23,3) \\
(43,3) \\
02 \\
01 \\
(13,3) \\
(3,3) \\
(10)\end{array}$ & $\begin{array}{c}10 \\
15 \\
(5,1) \\
(2,6) \\
05 \\
02 \\
04\end{array}$ & $\begin{array}{c}(12,8) \\
(5, I) \\
(10,3)\end{array}$ \\
\hline Total & 09 & $(100)$ & 30 & $(100)$ & 39 & $(100)$ \\
\hline
\end{tabular}

Qui-quadrado $p=0.439$ (não significante)

Tabela 3 - Distribuição de doentes portadores de condilomas anais agrupados conforme o padrão oncogênico (HPV 16, 18 e 3I) e não oncogênico (HPV 6 e II), comparados com o grau de neoplasia intra-epitelial anal (NIA). Instituto de Infectologia Emílio Ribas, São Paulo, 2003

\begin{tabular}{cccccc}
\hline Padrão do HPV & \multicolumn{2}{c}{ NIAA } & & \multicolumn{2}{c}{ NIAB } \\
\cline { 2 - 3 } \cline { 5 - 6 } & $n^{0}$ & $\%$ & & $n^{0}$ & $\%$ \\
não oncogênicos & 05 & 20 & & 20 & 80 \\
oncogênicos & 02 & 25 & & 06 & 75 \\
Total & $\mathbf{0 7}$ & $\mathbf{2 1 , 2}$ & & $\mathbf{2 6}$ & $\mathbf{7 8 , 8}$ \\
\hline
\end{tabular}

Qui-quadrado $p=1,000$ (não significante)

volume nuclear, hipercromasia e presença de figuras mitóticas restritas ao terço inferior do epitélio; displasia moderada, quando essas alterações acometiam os dois terços inferiores do epitélio; displasia acentuada, todo o epitélio envolvido, com perda da definição da camada basal ${ }^{6}$.

Adotamos para a displasia a denominação neoplasia intra-epitelial anal (NIA). Para os condilomas sem displasia e aqueles com displasia leve a classificação NIA de baixo grau de malignização (NIAB) e para os com displasias moderada e acentuada, NIA de alto grau de malignização (NIAA)".

O tipo viral foi identificado pelo método do PCR (reação em cadeia da polimerase) rotineiro para todos os tipos de HPV já conhecidos, a partir dos espécimes previamente fixados em formol e, no final, emblocados em parafina para estudo anatomopatológico. Realizamos cada corte com uma lâmina de micrótomo diferente para evitar contaminação viral. Embora tenhamos enviado material de 58 doentes, a presença do HPV só pode ser constatada em 39 deles.
Utilizamos o método do qui quadrado e teste exato de Fischer para análise estatís tica dos resultados, com nível de signi ficância de 5\%.

\section{Resultados}

Observamos NIAA em nove $(23,1 \%)$ e NIAB em 30 doentes (76,9\%). Os tipos virais mais observados encontram-se na Tabela I.

$\mathrm{Na}$ Tabela 2, mostramos o padrão histológico e os tipos virais. Na Tabela 3, observamos que tipos não oncogênicos do HPV provocaram NIAA. A análise estatística mostrou que tanto os tipos de HPV oncogênicos como os não oncogênicos estão relacionados ao aparecimento de NIAA nesse grupo de doentes.

\section{Discussão}

A região perianal é a área extra-genital mais freqüentemente acometida pelo HPV nos doentes HIV positivo ${ }^{20}$. Na literatura consultada, a incidência variou de $15,7 \%$ a $62 \% 10,21-23$. Podem ocorrer em qualquer faixa etária, entretanto, acometem principalmente adultos jovens, com o pico de incidência entre os $20 \mathrm{e}$ 24 anos $^{24}$, provavelmente quando a atividade sexual é maior.

Atualmente, há tendência ao agrupamento das lesões condilomatosas sem displasia e com displasia leve, ou seja, células com alterações nucleares restritas ao terço inferior do epitélio, sob o nome de NIAB e denominar as displasias moderada e acentuada, nas quais as modificações nucleares ocorrem nos dois terços inferiores ou em todo o epitélio, de NIAA". O objetivo é agrupar as lesões com comportamento semelhante. Devido a essa tendência, preferimos utilizar tal nomenclatura. Tivemos freqüência de NIAA em 23, 1 \% desse grupo de doentes, próxima à da literatura que observou entre $5,4 \%$ e $22 \%{ }^{11,25-27}$.

Os testes de escolha para detectar 0 tipo do HPV baseiam-se nas técnicas de identificação do ácido desoxidorribo nucleico (DNA). Têm sido utilizados o ensaio de captura híbrida (HCA) que distingue os tipos virais em oncogênicos e não oncogênicos sem os especificar, ${ }^{28}$ a hibridização "in situ" e a reação em cadeia da polimerase $(P C R)^{29}$, atualmente considerada o método mais eficaz ${ }^{29-31}$. Por esse motivo, foi teste de nossa escolha. 
Em nosso trabalho, encontramos $64 \%$ de tipos virais 6 e 11 e $8 \%$ dos tipos 16 e 18 nos portadores de condilomas anais. 0 HPVI 6 foi citado por alguns como o mais freqüente ${ }^{32,33}$. Não identificamos o tipo viral em quatro doentes, embora o teste reve lasse a presença do DNA viral. Talvez sejam novos tipos. Uma vez que o DNA isolado de tecido fixado tende a ser muito degradado, utilizou-se, alternativamente, oligonucleotídeos iniciadores capazes de amplificar fragmentos menores do genoma do HPV. Desta forma, garantiu-se a ausência de resultados falso-negativos.

A necessidade de se conhecer o tipo de HPV decorre da associação de alguns deles com lesões com alto grau de malignização 24,34,35. Todavia, em nosso estudo, não houve possibilidade de associar o padrão oncogênico do tipo de HPV com o grau da NIA, conforme relatado na literatura especializada $^{36-38}$. Mais importante foi a observação de que tipos não oncogênicos de HPV estavam associados à NIAA, fato antes não relatado pela literatura especializada. Devido a isso, sugerimos que os doentes HIV positivo com condilomas anais devam ter acompanhamento ambulatorial rigoroso, independente do tipo viral, pelo risco de desenvolver carcinomas.

Os resultados obtidos nas condições de execução deste estudo, em que avaliamos o tipo viral e o aspecto histológico das lesões, permitem concluir que tanto os tipos oncogênicos como os não oncogênicos de HPV podem estar associados com o desenvolvimento de NIA de alto grau em doentes HIV positivo.

\section{Conflito de interesse: não há.}

\section{SUMMARY}

\section{Human papillomavirus oncogenI- CITY AND GRADE OF ANAL INTRAEPITHELIAL NEOPLASIA IN HIV POSITIVE PATIENTS}

OBIECTIVE. We decided to evaluate if grade of anal intraepithelial neoplasia (AIN) was associated to the HPV type in HIV positive patients, since this group of patients has immunodeficiency over long periods. We identified HPV types by PCR (polimerase chain reaction) and histological examination to determine the AIN grade in $39 \mathrm{HIV}$ positive males with anal condylomata acuminata.
REsults. We observed high grade AIN (HAIN) in 9 (23. I percent) and low grade AIN (LAIN) in 30 patients (76.9 percent). The most frequent HPV types were 6 and II (64 percent) and oncogenictypes 16,18 and 31 appeared in 20.5 percent of patients. We could not identify viral type in four patients ( 10.2 percent) despite tests revealing presence of viral deoxiribonucleic acid. Comparing AIN grades with viral types we observed that non-oncogenic types can also be associated with HAIN lesions.

CONCLUSION. These results allowed us to conclude that both oncogenic and nononcogenic HPV types can be associated with HAIN lesions in HIV positive patients. [Rev Assoc Med Bras 2004; 50(3): 282-5]

KEY wORDS: AIDS. Human papillomavirus infection. Anal intraepithelial neoplasia. Polymerase chain reaction. Anal condylomata acuminata. Anal cancer.

\section{REFERÊNCIAS}

I. Wieland U, Pfister H. Papilloma virus in human pathology. In: Gross GE, Barrasso R, Human papilloma vírus infection: a clinical atlas. Berlin: Ullstein Mosby GmbH \& Co. KG; 1 997. p. I - 8.

2. Modesto VL, Gottesman L. Sexually transmitted diseases and anal manifestations of AIDS. Surg Clin North Am 1994; 74: I 433-64.

3. Fenger $C$. Anal canal tumors and their precursors. In: Rosen PP, Fechner RE, editors. Pathology annual. 1988, part I. Norwalk: Appleton and Lange; 1988. p.45-66.

4. Van Landuyt C, Mougin C, Drobacheff C, Bernard C, Merle C, Lab M, et al. Anogenital papillomavirus lesions in human with or without HIV infection. Comparison of colposcopic, histopathological and virological results. Ann Dermatolol Venereol 1993; 120:281-6.

5. Palefsky JM, Holly EA, Gonzales J, Lamborn K, Hollander $\mathrm{H}$. Natural history of anal cytologic abnormalities and papillomavirus infection among homosexual men with group IV HIV disease. J AIDS 1992; 5: 1258-65.

6. Carter PS, De Ruiter A, Whatrup C, Katzet DR, Ewings $P$, Mindel $A$, et al. Human immunodeficiency virus infection and genital warts as risk factors for anal intraepithelial neoplasia in homosexual men. Br J Surg 1995; 82:473-4.

7. Metcalf AM, Dean T. Risk of dysplasia in anal condyloma. Surgery 1995; I | 8:724-6.

8. Holly EA, Whittemore AS, Aston DA, Ahn DK, Nickoloff BJ, Kristiansen JJ. Anal cancer incidence: genital warts, anal fissure or fistula, hemorrhoids and smoking. J Natl Cancer Inst 1989; 81:|726-31

9. Palefsky JM, Holly EA, Gonzales J, Lamborn K, Hollander $\mathrm{H}$. Detection of human papillomavirus DNA in anal intraepithelial neoplasia and anal cancer. Cancer Res 1991; 51 : 1014-9.
10. Nadal SR, Manzione CR, Galvão VM, Salim VR, Speranzini MB. Perianal diseases in hivpositive patients compared with a seronegative population. Dis Colon Rectum 1999; 42:649-54.

II. Critchlow CW, Surawicz CM, Holmes KK, Kuypers J, Daling JR, Hawes SE, et al. Prospective study of high grade anal squamous intraepithelial neoplasia in a cohort of homosexual men: influence of HIV infection, immunosuppression and human papillomavirus infection. AIDS 1995; 9: I 255-62.

12. Kiviat N, Rompalo A, Bowden R, Galloway D, Holmes KK, Corey L, et al. Anal human papillomavirus infection among human immunodeficiency virus-seropositive and seronegative men. J Infect Dis 1990; 162:358-61.

13. Levy AJ. Anal carcinoma. In: Levy AJ. HIV infection and development of cancer. Washington DC.: ASM Press; 1994.

14. Xi LF, Critchlow CW, Wheeler CM, Koutsky LA, Galloway DA, Kuypers, J, et al. Risk of anal carcinoma in situ in relation to human papillomavirus type 16 variants. Cancer Res 1998; 58:3839-44.

15. Franco EL. Epidemiology of anogenital warts and cancer. Obstet Gynecol Clin North Am 1996; 23:597-623.

16. Euvrard $S$, Chardonnet $Y$. Lésions anales et pappilomavirus humains. Aspects virologiques, épidémiologiques et carcinologiques. Ann Dermatol Venereol 1991; I | 8:497-502.

17. Frisch M. On the etiology of anal squamous carcinoma. Dan Med Bull 2002; 49: 194-209.

18. Minkoff H, Ahdied L, Massad LS, Anastos K, Watts DH, Melnick S, et al. The effect of highly active antiretroviral therapy on cervical cytologic changes associated with oncogenic HPV among HIV-infected women. AIDS 200I; 15:2157-64.

19. Ahdied L, Klein RS, Burk R, Cu-Uvin S, Schuman P, Duerr A, et al. Prevalence, incidence, and type-specific persistence of human papillomavirus in human immunodeficiency virus (HIV)-positive and HIV-negative women. J Infect Dis 200 I; 184:682-90.

20. Mindel A, Carmody C. Anal and perianal lesions in adults. In: Gross GE, Barrasso R. Human papilloma virus infection: a clinical atlas. Berlin: Ullstein Mosby GmbH \& Co. KG; 1997. p.389-94.

21. Safavi A, Gottesman L, Dailey TH. Anorectal surgery in the HIV+ patient:update. Dis Colon Rectum 1991; 34:299-304.

22. Denis BJ, May T, Bigard MA, Canton P. Lésions anales et péri-anales au cours des infections symptomatiques par le $\mathrm{VIH}$. Étude prospective d'une série de 190 patients. Gastroenterol Clin Biol 1992; 16:148-54.

23. Orkin BA, Smith LE. Perianal manifestations of HIV infection. Dis Colon Rectum 1992; 35:310-14

24. Koutsky L. Epidemiology of genital human papillomavirus infection. Am J Med 1997: 102:3-8. 
25. Pera M, Sugranes G, Ordi J, Trias M. Asociación entre la infección por el virus del papiloma humano, las lesiones premalignas del cáncer anal y el virus de la inmunodeficiencia humana: estudio prospectivo en individuos con condilomas acuminados. Med Clin (Barc) 1999; 113:13-4.

26. Lacey HB, Wilson GE, Tilston P, Wilkins EG, Bailey AS, Corbitt G, et al. A study of anal intraepithelial neoplasia in HIV positive homosexual men. Sex Transm Infect 1999; 75:172-7.

27. Piketty C, Darragh TM, Da Costa M Bruneval P, Heard I, Kazatchkine MD, et al. High prevalence of anal human papillomavirus infection and anal cancer precursors among HIV-infected persons in the absence of anal intercourse. Ann Intern Med 2003; 1 38:453-9

28. Trofatter KF Jr. Diagnosis of human papillomavirus genital tract infection. Am J Med 1997; 102:21-7.

29. Lorincz AT. Molecular methods for the detection of human papillomavirus infection. Obstet Gynecol Clin North Am 1996; 23:707-30.
30. Delvenne P, Fontaine MA, Delvenne C, Nikkels A, Boniver J. Detection of human papillomaviruses in paraffin-embedded biopsies of cervical intraepithelial lesions: analysis by immunohistochemistry, in situ hybridization, and the polymerase chain reaction. Mod Pathol 1994; 7: I 13-9.

31. Surawicz CM, Critchlow C, Sayer J, Hurt C, Hawes $S$, Kirby $P$, et al. High grade anal dysplasia in visually normal mucosa in homosexual men: seven cases. Am J Gastroenterol 1995; 90: 1776-8.

32. Palefsky JM, Holly EA, Ralston ML, Da Costa M, Greenblatt RM. Prevalence and risk factors for anal human pappilomavirus infection in human immunodeficiency virus (HIV)-positive and high-risk HIV-negative women. J Infect Dis 2001; | 83:383-9|

33. Breese PL, Judson FN, Penley KA, Douglas JM Jr. Anal human papillomavirus infection among homosexual and bisexual men: prevalence of type-specific infection and association with human immunodeficiency virus. Sex Transm Dis 1995; 22:7-14.

34. Handsfield $\mathrm{HH}$. Clinical presentation and natural course of anogenital warts. Am J Med 1997; 102:16-20.
35. Unger ER, Vernon SD, Lee DR, Miller DL, Sharma S, Clancy KA, et al. Human papillomavirus type in anal epithelial lesions is influenced by human immunodeficiency virus. Arch Pathol Lab Med 1997; 121:820-4.

36. Lillo FB, Ferrari D, Veglia F, Origoni M, Lodini $S$, Mastrorilli E, et al. Human papillomavirus infection and associated cervical disease in human immunodeficiency virus-infected women: effect of highly active antiretroviral therapy. J Infect Dis 200 I; | 84:547-5 |.

37. Bjorge T, Engeland A, Luostarinen T, Mork J, Gislefoss RE, Jellum E, et al. Human papillomavirus infection as a risk factor for anal and perianal skin cancer in a prospective study. $\mathrm{Br}$ J Cancer 2002; 87:6I-4.

38. Frisch M, Glimelius B, Van der Brule AJ Wohlfahrt J, Meijer CJ, Walboomers JM, et al. Sexually transmitted infection as a cause of ana cancer. N Engl J Med 1997; 337: 1350-8.

Artigo recebido: 27/08/2003 Aceito para publicação: 09/03/2004 